\title{
cmaJOPEN
}

\section{Health care costs of hospitalization of young children for respiratory syncytial virus infections: a population-based matched cohort study}

\author{
Nisha Thampi MD MSc, Braden D. Knight MSc, Kednapa Thavorn PhD, Richard J. Webster PhD, \\ Krista Lanctot PhD, Steven Hawken PhD, J. Dayre McNally MD PhD
}

Abstract

Background: Respiratory syncytial virus (RSV) infection poses a substantial clinical burden among infants and young children. We sought to determine the health care costs of hospitalizations attributable to RSV in Ontario, Canada, from the health care payer perspective.

Methods: For this population-based matched cohort study, we identified children younger than 24 months who were or were not hospitalized with RSV infections in 2006-2016. We performed a cost-of-illness analysis using linked administrative health data, with subjects stratified by gestational age and congenital heart disease, and propensity score-matched on established risk factors. The primary outcome was attributable health care costs per patient, reflecting the difference in direct medical costs between the groups, calculated to 12 months postdischarge in 2020 Canadian dollars.

Results: We identified 14608 RSV-infected children, matched to 72040 controls. The adjusted attributable cost of hospitalized RSV was $\$ 134931900$ over 10 years, or $\$ 9240$ per patient (95\% confidence interval [Cl] \$8790-\$9690). Health care costs escalated 3 days before hospitalization, and persisted up to 12 months after discharge. Increased costs were associated with major comorbidities, but not extreme premature birth. The highest mean attributable cost per patient was in the presence of hemodynamically significant heart disease ( $\$ 60110,95 \%$ CI $\$ 26700-\$ 93060)$. Infants born at 36-43 weeks' gestation constituted the greatest overall cost burden at $\$ 117886720$.

Interpretation: Although the greatest direct medical costs per patient hospitalized with RSV infection are among children with cardiac disease, the greatest overall cost burden is from children born at or near term, who are not targeted by current prophylaxis strategies. The substantial attributable health care costs of RSV can inform cost-effectiveness analyses of novel RSV vaccines and prioritization of health care resources.

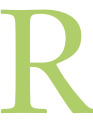
espiratory syncytial virus (RSV) is the most common respiratory viral pathogen among infants and young children, with 2 in 10 children needing urgent medical attention; almost all children get infected with RSV by 2 years of age. ${ }^{1-5}$ In Canada, $1 \%$ of newborns are hospitalized in the first year of life, with RSV infections accounting for $9 \%$ of all hospital admissions among infants for causes not related to birth. ${ }^{6}$ In addition to immediate consequences, RSV illness has been associated with long-term sequelae, including subsequent lower respiratory infection, asthma and sleep-disordered breathing. ${ }^{7,8}$

In the absence of a vaccine, the prevention of severe RSV disease has focused on population-based risk factor modification and administration of parenteral monoclonal RSV antibody to select high-risk groups (e.g., infants born prematurely, those with severe chronic lung disease and those with hemodynamically significant congenital heart disease)., ${ }^{2,9,10}$ However, rates of hospitalization for RSV infections have remained stable in the past decade, likely because fewer than $20 \%$ of children hospitalized with RSV meet the stringent eligibility criteria for palivizumab. ${ }^{6}$ The World Health Organization has prioritized RSV

Competing interests: Krista Lanctot reports grants from AbbVie Canada and stock from Highmark Interactive, outside the submitted work. J. Dayre McNally reports funding from the Canadian Institutes of Health Research, outside the submitted work. No other competing interests were declared.

This article has been peer reviewed.

Correspondence to: Nisha Thampi, nthampi@cheo.on.ca

CMAJ Open 2021 October 19. DOI:10.9778/cmajo.20200219 
vaccine development to address the global disease burden, particularly among low- and middle-income countries where palivizumab access is hindered by excessive costs and logistics. ${ }^{4,11}$ Therefore, there is an urgent need for new cost-effective measures to prevent and treat RSV infections.

Decision-makers require accurate estimates of costs attributable to disease to inform what prevention measures and treatments are economically viable. Operational decisions around RSV prevention and treatment programs, including novel vaccines, expanded palivizumab administration or targeted behavioural modification to high-risk groups, are affected by baseline data on disease costs. The reported mean cost per patient with RSV requiring hospitalization has ranged between $\$ 4400$ and $\$ 59000$ (in 2020 Canadian dollars) in Canada, the United States, the Netherlands and France. ${ }^{12-20}$ Those estimates vary, given differences in epidemiology, health care systems and payment mechanisms. ${ }^{18}$

The objective of this cost-of-illness study is to estimate the health care costs attributable to infants and young children hospitalized for RSV infection in Ontario, Canada, from the health care payer perspective. With vaccine development underway, an understanding of the immediate and long-term direct medical costs of severe RSV disease will help to inform prevention and treatment policies in public payer systems.

\section{Methods}

\section{Design and study population}

We conducted a population-based, retrospective matched cohort study of all children aged younger than 24 months in Ontario, Canada, to estimate the attributable health care costs of severe RSV illness. We included children born between Apr. 1, 2006, and Mar. 31, 2016, who were covered continuously by the provincial health insurance plan from birth until 3 years of age. Our study used routinely collected administrative health databases housed at ICES (Table 1). ICES is an independent, nonprofit research institute whose legal status under Ontario's health information privacy law allows it to collect and analyze health care and demographic data, without consent, for health system evaluation and improvement.

\section{Exposure}

The primary exposure was hospitalization for severe RSV illness in the first 24 months of life. We identified hospitalizations from the Discharge Abstract Database, compiled by the Canadian Institute for Health Information, using a validated algorithm of RSV-associated codes from the International Classification of Diseases, 10th Revision (Appendix 1, Table S1, available at www.cmajopen.ca/content/9/4/E948/suppl/ DC1). ${ }^{6}$ This algorithm is accurate in identifying RSV admissions using administrative health data, with $97.9 \%$ sensitivity and $96.9 \%$ positive predictive value. ${ }^{6}$

We defined a case as a child admitted to a health care facility in Ontario with a diagnosis of RSV infection within their first 24 months of life; controls were children residing in Ontario who were never hospitalized with a diagnosis of $\mathrm{RSV}$ infection in the same period. As it was not possible to calculate attributable costs for children with RSV infections who died within a year of their index date (defined below), we instead reported cumulative costs for this group.

\section{Outcomes}

The primary outcome was attributable, direct medical costs per child hospitalized with RSV over a 12 -month period. We performed analyses from the perspective of the public health care payer and included health care costs borne to the Ontario Ministry of Health (Ministry of Health and LongTerm Care during the study period) (i.e., direct medical costs). We used a person-level costing algorithm developed and validated at ICES. The methods have been previously described in detail. ${ }^{21}$

Briefly, we assessed total health system costs per patient by summing each single cost component that contributed to the provision of the service (Appendix 1, Table S2). We estimated the costs of inpatient hospitalizations, emergency department visits, same-day surgeries and inpatient rehabilitation by multiplying the weighted volume of services by the average provincial costs per weighted case. We obtained the costs of physician and outpatient diagnostic or laboratory services from an approved fee schedule as outlined in the Ontario Health Insurance Schedule of Benefits and Fees. For physicians using payment models other than fee-for-service, we calculated physician payments by applying applicable capitation payments or the median amount reimbursed for the same service code for the specific fiscal year. We estimated costs for highcost medical equipment from the amount reimbursed to

\begin{tabular}{|c|c|}
\hline Category & Database \\
\hline Care providers & ICES Physician Database \\
\hline \multirow[t]{3}{*}{ Coding and geography } & Drug Identification Numbers \\
\hline & Local Health Integration Network \\
\hline & Postal Code Conversion File \\
\hline Facilities & $\begin{array}{l}\text { Institutions funded by the Ministry of } \\
\text { Health }\end{array}$ \\
\hline \multirow[t]{2}{*}{ Financial } & Average Price of Health Services \\
\hline & Estimated Schedule of Benefits \\
\hline \multirow[t]{9}{*}{ Health services } & Continuing Care Reporting System \\
\hline & Discharge Abstract Database \\
\hline & Home Care Database \\
\hline & $\begin{array}{l}\text { National Ambulatory Care Reporting } \\
\text { System }\end{array}$ \\
\hline & $\begin{array}{l}\text { National Rehabilitation Reporting } \\
\text { System }\end{array}$ \\
\hline & Ontario Drug Benefit Claims \\
\hline & Ontario Health Insurance Plan \\
\hline & $\begin{array}{l}\text { Ontario Mental Health Reporting } \\
\text { System }\end{array}$ \\
\hline & Same Day Surgery \\
\hline
\end{tabular}


patients recorded in the Assistive Devices Program database. Costs of complex continuing care were based on case mix, number of days in care and resource utilization groups. We used pharmacy payments recorded in the Ontario Drug Benefit database to capture prescription medication costs for individuals eligible for public coverage.

We analyzed data sets at ICES, linked using unique encoded identifiers. We used index dates for patients with RSV and matched controls to determine the cost window. Costs related to palivizumab administration were not available.

For patients, we used the admission date for their first RSV hospitalization as the index date. As controls were not hospitalized for RSV, we randomly generated their index dates based on the distribution of index dates among patients with RSV. Recognizing that children with RSV often receive medical care before hospitalization, we visually inspected the data to determine the prehospitalization start date for the cost window. The time horizon was 12 months after the index date, in keeping with previous economic analyses, with the aim of capturing costs associated with severe RSV disease and sequelae. ${ }^{16,18,22}$ Once index dates were established, we identified relevant covariates, which were used in propensity score matching analysis.

\section{Statistical analysis}

To minimize potential unmeasured confounding factors, we initially stratified patients with RSV and controls by the presence of major risk factors, namely hemodynamically significant congenital heart disease and gestational age groups, using published algorithms of diagnostic codes. ${ }^{6}$ Once stratified into these subgroups, we matched patients with up to 5 controls by birth month, year and propensity score, using a caliper of 0.2 standard deviation (SD) of the propensity score. ${ }^{23}$

We employed a propensity score to balance covariates between patients and controls, and therefore minimize bias when calculating attributable costs. We included the following covariates: sex, regional health network, rurality, income quintile, maternal age, size for gestational age, birth order, twin status, chronic lung disease, trisomy 21 and complex chronic condition categories (Appendix 1, Table S3). Their selection was guided by existing literature and the opinions of clinical experts (N.T., K.L., J.D.M.) based on the RSV risk scoring tool and provincial indications for palivizumab, and was subject to data availability. ${ }^{24}$ For identification of complex chronic conditions, we used the framework established by Feudtner and colleagues and previously adapted to the Canadian context, although we treated cardiac categories, used to identify congenital heart disease and trisomy 21 , as separate covariates. ${ }^{25,26} \mathrm{We}$ used the standardized mean differences in covariates and distributions of propensity scores to check the balance in the matched sets.

We attributed the difference in mean total costs between patients with RSV and matched controls to RSV, assuming all other factors being equal from the propensity score matching process. We calculated confidence intervals (CIs) for the mean difference of percentiles 2.5 and 97.5 of 1000 bootstrap replications, resampling matched sets of patients and controls.
We calculated costs in 2017 Canadian dollars and adjusted them to 2020 Canadian dollars (2017 \$1 = 2020 \$1.05), and stratified across age groups. ${ }^{12-14}$

\section{Ethics approval}

This study was approved by the Research Ethics Board of the CHEO Research Institute in Ottawa. ICES is a prescribed entity under section 45 of Ontario's Personal Health Information Protection Act. Section 45 authorizes ICES to collect personal health information, without consent, for the purpose of analysis or compiling statistical information with respect to the management, evaluation or monitoring of the allocation of resources to or planning for all or part of the health system. Projects conducted under section 45 , by definition, do not require review by a research ethics board. This project was conducted under section 45, and approved by ICES' Privacy and Legal Office.

\section{Results}

During the study period, 1340330 children were born in Ontario, from which we identified 14790 children hospitalized with RSV. The crude mean annual incidence rate of hospitalized RSV was 6.38 per 1000 children (95\% CI 6.286.48 ) or 8.64 per 1000 person-years (95\% CI 8.51-8.78), with no significant trend over time (Appendix 1, Table S4). The median age at hospitalization was 3 (interquartile range [IQR] 1-8 ) months. The median length of index hospitalization stay was 3 (IQR 2-4) days. Twenty patients died during hospitalization and another 31 died within the follow-up period.

We excluded 145 (<1\%) patients and 18084 (1.5\%) controls from the cohort because they did not have provincial health insurance coverage for the full 12 months following their RSV-associated admission. Of the eligible cases, we could not match $131(0.9 \%)$ with at least 1 control, and we therefore excluded them from the cost analysis. After we applied exclusion criteria and propensity score matching, the final cohort size was 14608 children with RSV and 72040 matched controls (Figure 1). Unmatched cases were more likely to be premature, small for gestational age and have multiple comorbidities (Appendix 1, Table S5).

No meaningful imbalances between patients and their matched controls existed with respect to variables included in the propensity score after matching (i.e., all standardized differences were less than 0.1; Appendix 1, Table S6). Patients and controls had a similar propensity score distribution (Appendix 1, Figure S1).

\section{Attributable costs of RSV}

We observed an escalation of costs no earlier than 3 days before hospitalization; therefore, we calculated costs starting from 7 days before the index date (Appendix 1, Figure S2). The hospitalization period represented $59 \%$ of total costs, followed by the postdischarge period (37\%). The mean cost per patient with RSV requiring hospitalization was $\$ 12080$ (SD \$28 229), compared with \$2410 (SD \$16 305) for matched controls (Table 2). Over the 10-year period, the adjusted attributable cost of children under 24 months who 


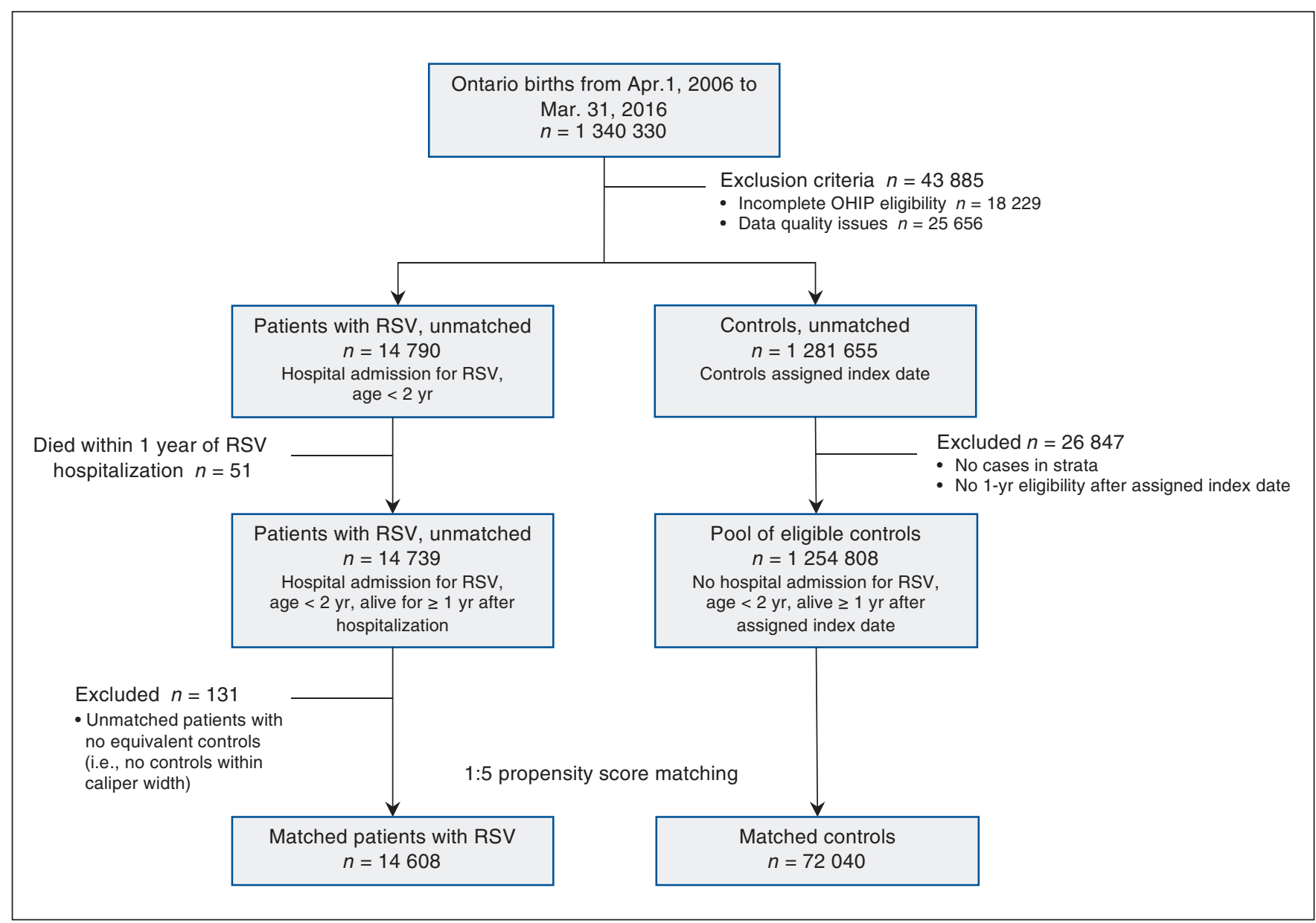

Figure 1: Identification of patients hospitalized with respiratory syncytial virus (RSV) and controls. Patients were matched with up to 5 controls, where possible. Ideally, 5 matches were found per case; in the event of fewer matches, a lower matching ratio was permitted.

were hospitalized with RSV was \$134931900 (Appendix 1, Table S7), or $\$ 9240$ per patient (95\% CI $\$ 8790-\$ 9690)$ (Table 2). The annual attributable cost of RSV requiring hospitalization ranged between $\$ 10.4$ million and $\$ 16.0$ million (Appendix 1, Table S7).

Figure 2 illustrates the cost distribution per individual, showing higher expenditure in cases compared with matched controls. Patients with known risk factors for RSV (e.g., congenital heart disease, chronic lung disease and prematurity) had significantly increased attributable health care costs; the highest cost per case was associated with hemodynamically significant congenital heart disease at \$60 110 (95\% CI \$26 700 $\$ 93$ 060; Table 2). Infants born at 36-43 weeks' gestation comprised $90 \%$ of all hospitalized patients. This gestational age group had the lowest attributable cost per patient at $\$ 8920$, but reflected the greatest overall cost burden at an average annual attributable cost of $\$ 11.8$ million. There were no significant cost differences across age groups at diagnosis.

All cost categories were significantly higher $(p<0.001)$ for patients compared with controls (Appendix 1, Figure S3), with the major difference driven by costs during the index hospitalization. Patients with RSV also had higher costs from physician billings and emergency department visits in the period leading up to and including hospitalization (average cost per patient $\$ 1766$ and $\$ 1050$, or $14.7 \%$ and $8.8 \%$ of total costs, respectively). There were no differences in costs per patient among hospitalized children who lived in urban (\$12 150) versus rural settings ( $\$ 11400, p=0.295$ ). Differences in average costs were most notable in the first 4 months and persisted to 12 months after discharge, with the greatest cost differences for inpatient and hospital outpatient care and physician visits in the first month (Figure 3).

There were substantial overall direct medical costs associated with RSV illness among patients who died during the study period or were unmatched (Appendix 1, Table S8). The 1-year total health care cost for unmatched patients with RSV was $\$ 15468530$ (average $\$ 118080$ per patient). The total cost of patients who died during hospitalization was \$1 767250 (average \$88 360 per patient).

\section{Interpretation}

In an Ontario birth cohort (2006-2016), nearly 15000 children were hospitalized for severe RSV before age 24 months, with the greatest burden under 6 months of age, consistent with the global epidemiology of severe RSV. ${ }^{4}$ We used 


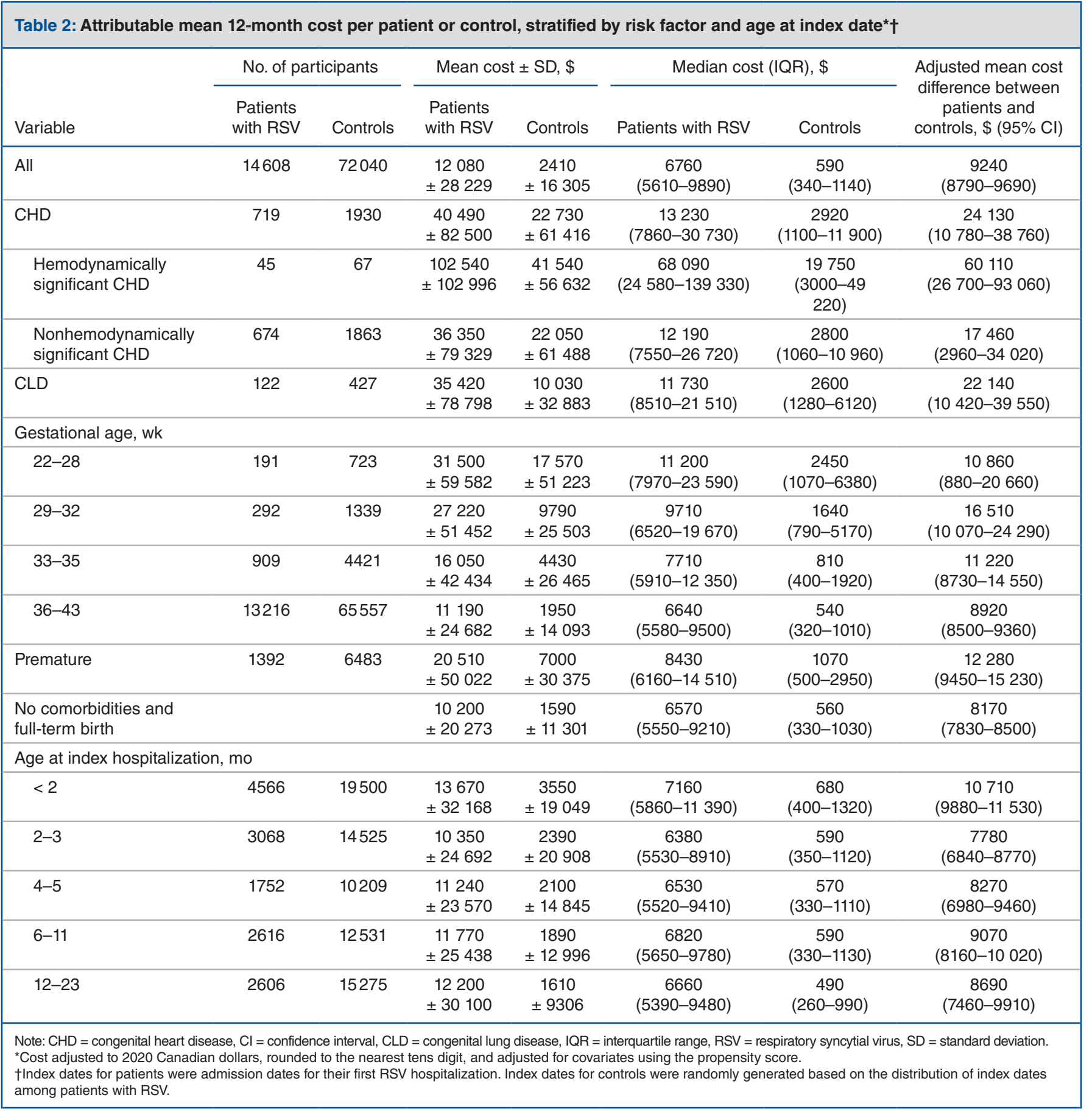

a previously validated algorithm to identify admissions for RSV in the cohort of 1.34 million newborns accurately. ${ }^{6}$ During this study period, the prevalence of RSV infections did not change significantly, particularly among those ineligible for palivizumab after its introduction in 2002 for at-risk patient populations. ${ }^{6}$ The mean attributable cost per patient hospitalized with RSV was \$9240 (95\% CI \$8790-\$9690). Higher costs per patient were observed in high-risk groups, particularly among children with hemodynamically significant heart disease; however, the greatest burden was among infants born between 36 and 43 weeks' gestation, at an average annual cost of $\$ 11.8$ million.

Respiratory syncytial virus requiring hospitalization has been associated with substantial health care costs, influenced by age, degree of prematurity, presence of comorbid conditions, duration of hospitalization and rurality. ${ }^{15-18,22,27}$ The mean cost estimate for children with RSV aged younger than 5 years in Ontario was recently reported at $\$ 4150$ (2015 dollars) per patient, with no difference by presence of a comorbid condition. ${ }^{1}$ That figure reflected overall costs associated 


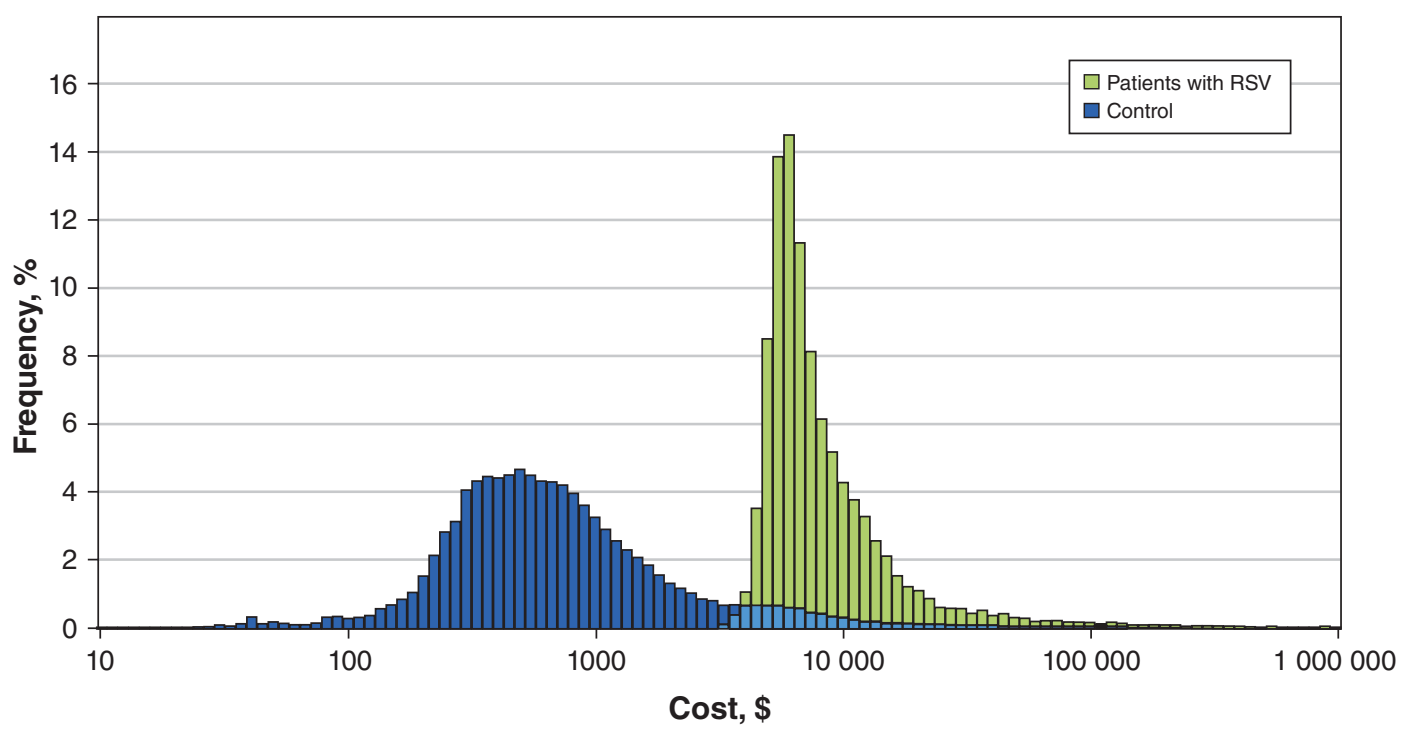

Figure 2: Frequency of cost per individual, compared between patients hospitalized with respiratory syncytial virus (RSV) and controls. Costs are represented on a logarithmic scale (2020 Canadian dollars).

with RSV, and excluded attributable costs such as emergency department visits, outpatient visits and costs after discharge. A similar methodological approach among infants hospitalized with RSV in France reported a mean cost per hospitalized child of $€ 3793$ (about Can\$6200); direct medical costs of children aged younger than 2 years who were hospitalized with RSV in the Netherlands were similar to our estimates. ${ }^{15,19}$ The overall costs of extremely premature infants hospitalized with RSV in the US were 4 times higher per patient than term infants. ${ }^{16}$ In contrast, we found only a $22 \%$ difference in attributable costs between these groups. Although most costs were associated with hospitalization, we observed additional costs in the 3 days before admission and up to 12 months after discharge. The use of propensity score weighting enabled more accurate determination of costs by controlling for confounding factors, such that costs unrelated to the health problem were not incorrectly attributed.

Among economic evaluations performed in the Canadian context, 3 key differences have limited the generalizability of cost estimates: setting, with rural transportation a major cost driver in 1 study; epidemiology, with broad variability in risk factors and disease incidence among northern communities, patients with cystic fibrosis, infants with hemodynamically significant congenital heart disease, and infants born at 32-35 weeks' gestation; and costing methodology, from the use of hospital rates to provincial costing methodologies, with no studies evaluating posthospitalization costs with a matched cohort of children. ${ }^{28-33}$

The attributable costs of RSV reported in this study will enable researchers to estimate the potential economic viability of palivizumab prophylaxis and future RSV vaccines. Despite the substantial global burden of disease, no RSV vaccines are currently approved for pregnant women or infants. ${ }^{4}$ Multiple protective strategies have been suggested, including maternal cocooning and infant vaccination, to target young infants at highest risk for severe disease. ${ }^{34}$ The World Health Organization has identified RSV vaccine development as a research priority.

\section{Limitations}

The average annual cost of hospitalized RSV in Ontario, at $\$ 13.5$ million, is likely a conservative estimate. Our analysis excluded RSV-related deaths and patients with RSV and multiple comorbidities who could not be matched to controls; however, together they accounted for $1.2 \%$ of the total cohort of patients hospitalized with RSV. Unmatched cases had substantial overall costs of care that would be expected to increase the cost-of-illness estimate, albeit with uncertainty as to whether the costs could be attributed to the acute illness or to highly complex medical conditions. ${ }^{35}$

Although the focus of the study was on direct medical costs of severe RSV requiring hospitalization, less severe RSV, requiring visitation to family physicians or emergency departments, also creates direct medical costs that were not calculated in this study. No validated algorithm exists to identify this patient cohort, and urgent care visits would reflect much lower costs than hospitalizations.

A recent study showed increased health care resource utilization for 5 years after infant RSV infection compared with healthy controls, although the greatest difference in burden was in the first 2 years. ${ }^{36}$ We were unable to include out-of-pocket patient costs, including caregiver time, transportation and loss of productivity during children's illness periods, which would suggest these figures are an underestimate of the societal costs. Moreover, we could not account for transportation costs borne to the 


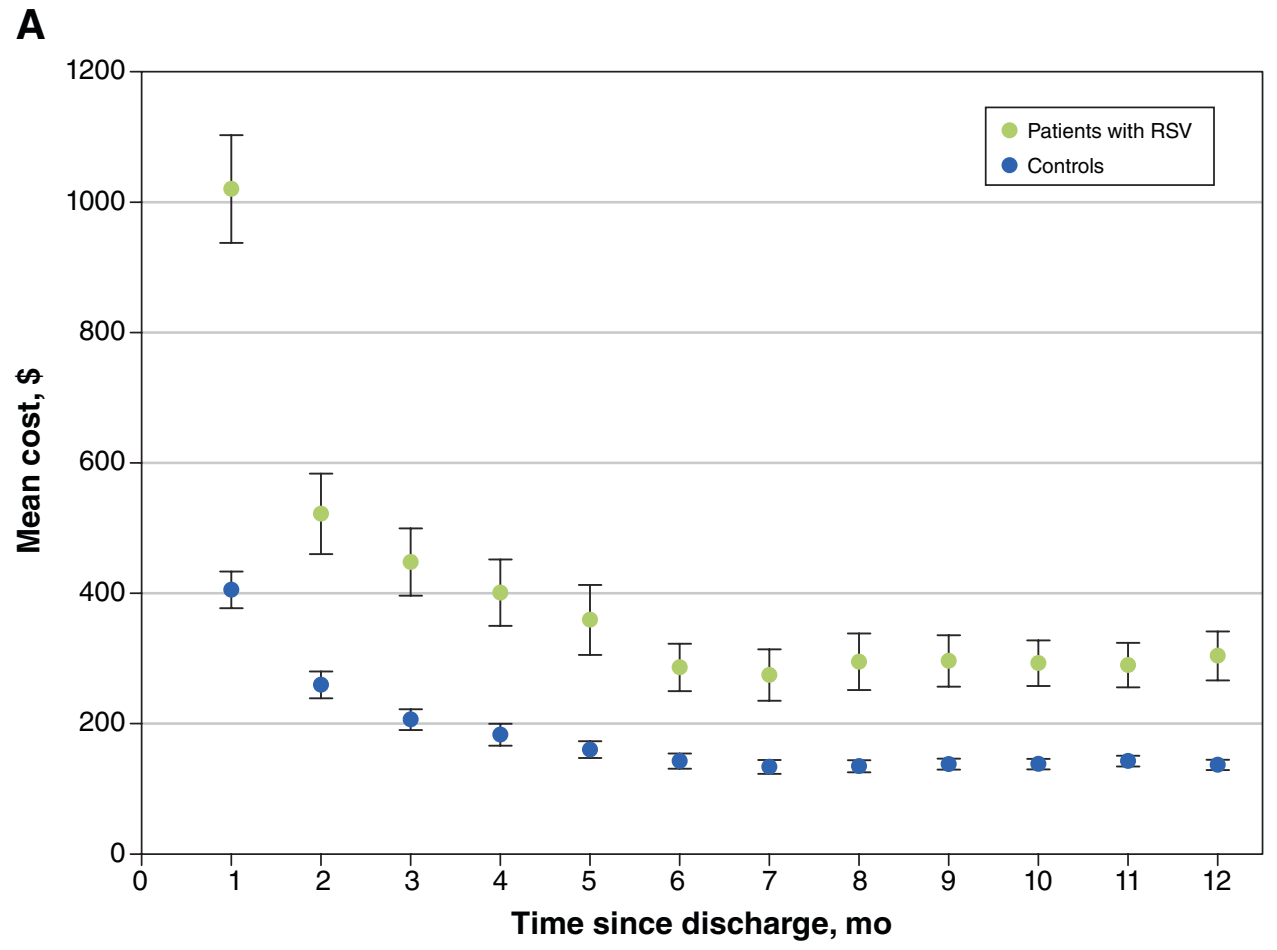

B

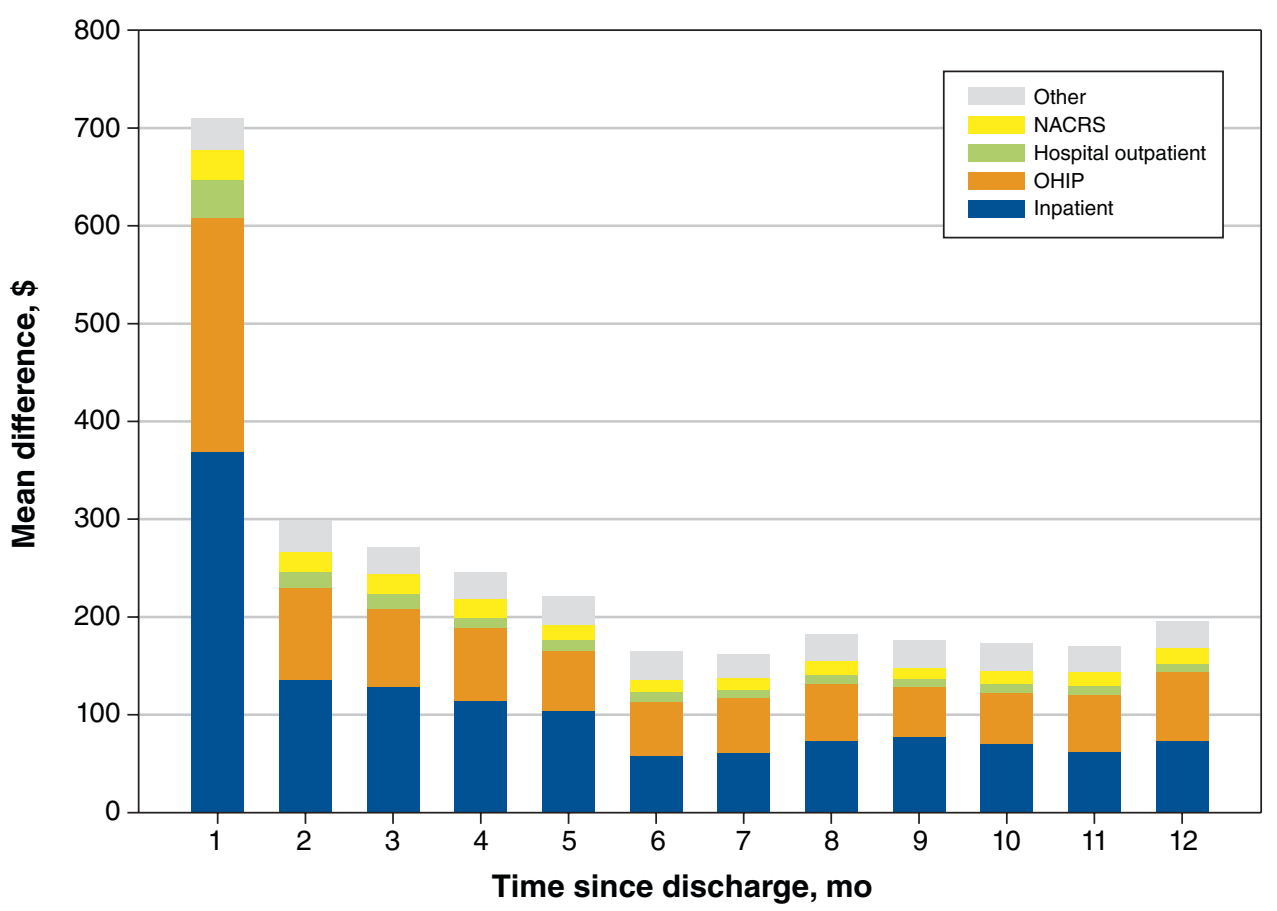

Figure 3: Cost over time between patients hospitalized with respiratory syncytial virus (RSV) and controls (2020 Canadian dollars). (A) Monthly mean costs per patient compared with control after discharge. (B) Monthly mean cost differences after discharge between patients and controls, stratified by cost component. Note: NACRS = National Ambulatory Care Reporting System, OHIP = Ontario Health Insurance Plan. "Other" costs include those attributed to the Ontario Drug Benefit, complex \& continuing care, home care, capitation for family health organizations or networks and the Assistive Devices Program. 
public payer, which is a substantial driver of costs for patients in remote Canadian communities who require transfer of care from their regional centres to specialized pediatric centres. ${ }^{37}$

We were unable to differentiate between communityassociated and health care-associated RSV infections. However, the incidence of health care-associated RSV in Ontario is $4 \% .{ }^{1}$ Although we sought to address confounding through propensity score matching, this analytic approach allows adjustment only for observed confounding factors. Unmeasured confounders associated with infant fragility (e.g., smoking in the home, daycare, genetic susceptibility) may further modify differences between these groups.

Finally, we were unable to link and evaluate the impact of palivizumab on health care costs at the individual level. However, most hospitalized infants in our study would not have qualified for prophylaxis by strict eligibility criteria, similar to other studies showing substantial health care resource use, morbidity and death in the absence of known risk factors for severe RSV. ${ }^{2,35,38}$ Despite these limitations, our conservative estimates are concerning enough to urge policy-makers to examine new strategies that will reduce the incidence and economic burden of RSV disease among young children.

\section{Conclusion}

In this study, we evaluated the attributable incident costs of severe RSV disease in a large, rigorously matched cohort of infants and young children in Ontario, Canada. At an attributable cost of $\$ 9240$ per patient, this cost-of-illness analysis provides muchneeded data on direct health care costs attributable to hospitalized RSV in the largest published cohort of children under 2 years of age. As RSV vaccine development is a global research priority, our data will enable decision-makers in a public payer system to compare new interventions and judiciously allocate health care resources to prevent severe RSV in the very young.

\section{References}

1. Buchan SA, Chung H, Karnauchow T, et al. Characteristics and outcomes of young children hospitalized with laboratory-confirmed influenza or respiratory syncytial virus in Ontario, Canada, 2009-2014. Pediatr Infect Dis 7 2019;38:362-9

2. Hall CB, Weinberg GA, Iwane MK, et al. The burden of respiratory syncytial virus infection in young children. N Engl 7 Med 2009;360:588-98.

3. Buchwald AG, Tamboura B, Tennant SM, et al. Epidemiology, risk factors, and outcomes of respiratory syncytial virus infections in newborns in Bamako, Mali. Clin Infect Dis 2020;70:59-66.

4. Shi T, McAllister DA, O'Brien KL, et al.; RSV Global Epidemiology Network. Global, regional, and national disease burden estimates of acute lower respiratory infections due to respiratory syncytial virus in young children in 2015: a systematic review and modelling study. Lancet 2017;390:946-58.

5. Hall CB, Weinberg GA, Blumkin AK, et al. Respiratory syncytial virusassociated hospitalizations among children less than 24 months of age. Pediatrics 2013;132:e341-8.

6. Pisesky A, Benchimol EI, Wong CA, et al. Incidence of hospitalization for respiratory syncytial virus infection amongst children in Ontario, Canada: a population-based study using validated health administrative data. PLoS One 2016;11:e0150416.

7. Falsey AR, McElhaney JE, Beran J, et al. Respiratory syncytial virus and other respiratory viral infections in older adults with moderate to severe influenza-like illness. 7 Infect Dis 2014;209:1873-81.

8. Hyvärinen M, Piippo-Savolainen E, Korhonen K, et al. Teenage asthma after severe infantile bronchiolitis or pneumonia. Acta Paediatr 2005;94:1378-83.

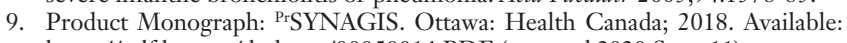
https://pdf.hres.ca/dpd_pm/00050014.PDF (accessed 2020 Sept. 11).

10. Palivizumab, a humanized respiratory syncytial virus monoclonal antibody, reduces hospitalization from respiratory syncytial virus infection in high-risk infants. The IMpact-RSV Study Group. Pediatrics 1998;102:531-7.
11. RSV vaccine research and development technology roadmap: Priority activities for development, testing, licensure and global use of RSV vaccines, with a specific focus on the medical need for young children in low- and middle-income countries. Geneva: World Health Organization; 2017.

12. Exchange rates. Ottawa: Bank of Canada. Available: https://www.bankofcanada .ca/rates/exchange (accessed 2021 Jan. 29).

13. Inflation Calculator. Ottawa: Bank of Canada. Available: https://www. bankofcanada.ca/rates/related/inflation-calculator/ (accessed 2021 Jan. 28).

14. Inflation Calculator CPI. Washington (DC): U.S. Bureau of Labor Statistics. Available: https://www.bls.gov/data/inflation_calculator.htm (accessed 2021 Jan. 29).

15. Kramer R, Duclos A, VRS study group in Lyon, et al. Cost and burden of RSV related hospitalisation from 2012 to 2017 in the first year of life in Lyon, France. Vaccine 2018;36:6591-3.

16. McLaurin KK, Farr AM, Wade SW, et al. Respiratory syncytial virus hospitalization outcomes and costs of full-term and preterm infants. $\mathcal{F}$ Perinatol 2016;36:990-6.

17. Meijboom MJ, Rozenbaum MH, Benedictus A, et al. Cost-effectiveness of potential infant vaccination against respiratory syncytial virus infection in The Netherlands. Vaccine 2012;30:4691-700.

18. Palmer L, Hall CB, Katkin JP, et al. Healthcare costs within a year of respiratory syncytial virus among Medicaid infants. Pediatr Pulmonol 2010;45:772-81.

19. Rietveld E, De Jonge HCC, Polder JJ, et al. Anticipated costs of hospitalization for respiratory syncytial virus infection in young children at risk. Pediatr Infect Dis 7 2004;23:523-9.

20. Ledbetter J, Brannman L, Wade SW, et al. Healthcare resource utilization and costs in the 12 months following hospitalization for respiratory syncytial virus or unspecified bronchiolitis among infants. F Med Econ 2020;23:139-47.

21. Wodchis WP, Bushmeneva K, Nikitovic M, et al. Guidelines on person-level costing using administrative databases in Ontario. Toronto: University of Toronto Libraries; 2013. Available: https://tspace.library.utoronto.ca/handle /1807/87373 (accessed 2020 Sept. 11).

22. Shi N, Palmer L, Chu B-C, et al. Association of RSV lower respiratory tract infection and subsequent healthcare use and costs: a Medicaid claims analysis in early-preterm, late-preterm, and full-term infants. 7 Med Econ 2011;14. $335-40$.

23. Rassen JA, Shelat AA, Myers J, et al. One-to-many propensity score matching in cohort studies. Pharmacoepidemiol Drug Saf 2012;21(Suppl 2):69-80.

24. Sampalis JS, Langley J, Carbonell-Estrany X, et al. Development and validation of a risk scoring tool to predict respiratory syncytial virus hospitalization in premature infants born at 33 through 35 completed weeks of gestation. Med Decis Making 2008;28:471-80.

25. Feudtner C, Feinstein JA, Zhong W, et al. Pediatric complex chronic conditions classification system version 2: updated for ICD-10 and complex medical technology dependence and transplantation. BMC Pediatr 2014;14:199.

26. McKelvie B, McNally JD, Chan J, et al. Increased mortality and length of stay associated with medical emergency team review in hospitalized pediatric patients: a retrospective cohort study. Pediatr Crit Care Med 2017;18:571-9.

27. Hasegawa K, Tsugawa Y, Brown DFM, et al. Trends in bronchiolitis hospitalizations in the United States, 2000-2009. Pediatrics 2013;132:28-36.

28. Banerji A, Ng K, Moraes TJ, et al. Cost-effectiveness of palivizumab compared to no prophylaxis in term infants residing in the Canadian Arctic. CMA7 Open 2016;4:E623-33.

29. Harris KC, Anis AH, Crosby MC, et al. Economic evaluation of palivizumab in children with congenital heart disease: a Canadian perspective. Can 7 Cardiol 2011;27:523.e11-5.

30. McGirr AA, Schwartz KL, Allen U, et al. The cost-effectiveness of palivizumab in infants with cystic fibrosis in the Canadian setting: a decision analysis model. Hum Vaccin Immunother 2017;13:599-606.

31. Smart KA, Paes BA, Lanctôt KL. Changing costs and the impact on RSV prophylaxis. 7 Med Econ 2010;13:705-8.

32. Tam DY, Banerji A, Paes BA, et al. The cost effectiveness of palivizumab in term Inuit infants in the Eastern Canadian Arctic. F Med Econ 2009;12:361-70.

33. Mac S, Sumner A, Duchesne-Belanger S, et al. Cost-effectiveness of palivizumab for respiratory syncytial virus: a systematic review. Pediatrics 2019;143:e20184064.

34. Cromer D, van Hoek AJ, Newall AT, et al. Burden of paediatric respiratory syncytial virus disease and potential effect of different immunisation strategies: a modelling and cost-effectiveness analysis for England. Lancet Public Health 2017;2:e367-74.

35. Tam J, Papenburg J, Fanella S, et al. Pediatric Investigators Collaborative Network on Infections in Canada study of respiratory syncytial virus-associated deaths in pediatric patients in Canada, 2003-2013. Clin Infect Dis 2019;68:113-9.

36. Simões EAF, Chirikov V, Botteman M, et al. Long-term assessment of healthcare utilization five years after respiratory syncytial virus infection in US infants. 7 Infect Dis 2020;221:1256-70.

37. Banerji A, Lanctôt KL, Paes BA, et al. Comparison of the cost of hospitalization for respiratory syncytial virus disease versus palivizumab prophylaxis in Canadian Inuit infants. Pediatr Infect Dis 7 2009;28:702-6.

38. Anderson EJ, DeVincenzo JP, Simões EAF, et al. SENTINEL1: two-season study of respiratory syncytial virus hospitalizations among U.S. infants born at 29 to 35 weeks' gestational age not receiving immunoprophylaxis. Am 7 Perinatol 2020;37:421-9. 
Affiliations: Department of Pediatrics (Thampi, McNally), Faculty of Medicine, University of Ottawa; CHEO Research Institute (Thampi, McNally); ICES uOttawa (Knight); Ottawa Hospital Research Institute (Knight, Thavorn); Clinical Research Unit, CHEO Research Institute (Knight, Webster); Ontario Child Health Support Unit (Knight, Webster); School of Epidemiology and Public Health (Thavorn, Hawken), University of Ottawa, Ottawa, Ont.; ICES Central (Thavorn, Hawken), Toronto, Ont.; Clinical Epidemiology Program (Thavorn), Ottawa, Ont.; Sunnybrook Research Institute (Lanctot), University of Toronto, Toronto, Ont.; Clinical Epidemiology Program (Hawken), Ottawa Hospital Research Institute, Ottawa, Ont.

Contributors: Nisha Thampi, Braden Knight, Kednapa Thavorn, Richard Webster and J. Dayre McNally designed the study, and collected, analyzed and interpreted the data. Nisha Thampi and Braden Knight wrote the first draft; Kednapa Thavorn, Richard Webster, Krista Lanctot, Steven Hawken and J. Dayre McNally reviewed the data and revised the report. All of the authors gave final approval of the version to be published and agreed to be accountable for all aspects of the work.

Funding: Braden Knight received support from the Ontario Child Health Support Unit, which is funded by the Ontario SPOR SUPPORT Unit and supported by the Canadian Institutes of Health Research and the Province of Ontario, which had no role in the study design, collection, analysis and interpretation of the data, writing of the report or decision to submit the manuscript for publication.

Content licence: This is an Open Access article distributed in accordance with the terms of the Creative Commons Attribution (CC BY-NC-ND 4.0) licence, which permits use, distribution and reproduction in any medium, provided that the original publication is properly cited, the use is noncommercial (i.e., research or educational use), and no modifications or adaptations are made. See: https://creativecommons.org/licenses/by-nc-nd/4.0/

Data sharing: The data used in this study were obtained from ICES, and the authors no longer have access to these data. As such, the data are not available for use by other researchers, other than what is presented in the manuscript.

Disclaimer: This study was supported by ICES, which is funded by an annual grant from the Ontario Ministry of Health $(\mathrm{MOH})$. Parts of this material are based on data and information compiled and provided by $\mathrm{MOH}$ and the Canadian Institute for Health Information. The analyses, conclusions, opinions and statements expressed herein are solely those of the authors and do not reflect those of the funding or data sources; no endorsement is intended or should be inferred.

Supplemental information: For reviewer comments and the original submission of this manuscript, please see www.cmajopen.ca/content/9/4/ E948/suppl/DC1. 